ISNN 0001-6002 /97/39/2/38-43

ORIGINAL

Acta Médica Costarricense, $\odot 1997$

Colegio de Médicos y Cirujanos

\title{
La mortalidad en el cuerpo médico nacional
}

\author{
Manuel Fco. Jiménez Navarrete
}

\section{Resumen}

Justificación: El médico es un profesional expuesto a la aparición temprana de morbimortalidad por varias enfermedades como. producto de su trabajo. En nuestro país debe verificarse esta afirmación.

Objetivo: Investigar la mortalidad en los últimos once años, en médicos costarricenses, mediante un estudio transversal descriptivo.

Métodos: Análisis bioestadístico de médicos fallecidos, según Métodos: Análisis bioestadístico del Colegio de Médicos y Cirujanos, Registro Civil y tres instituciones de salud. Se analizaron de los médicos vivos, su número, condición de actividad, sexo, edad, domicilio, lugar de formación, especialidad y actividad laboral. De los fallecidos, especialidad, edad al morir y sexo, causas primarias y secundarias de defunción.

Resultados: Había en Costa Rica 3894 médicos (1991), la mayoría especialistas $(43,6 \%)$, una proporción $3: 1$ (hombres/mujeres), formados en el país principalmente $(58,9 \%)$, la mayoría menores de 40 años de edad, viviendo en San José $(66,2 \%)$ e institucionalmente trabajando para la Caja Costarricense de Seguro Social (80\%). Durante el tiempo estudiado fallecieron 119 médicos (95\% hombres), principalmente especialistas $(56,4 \%)$, la mayoría entre 60 y 79 años de edad (40\%).

La principal causa de muerte fue la cardiovascular $(39,2 \%)$ destacando la enfermedad coronaria, produciendose hasta en un $54.5 \%$ entre los médicos activos.

La segunda causa de muerte fueron los carcinomas en un $20.5 \%$

Se encontró un $10 \%$ de suicidios y entre las causas infecciosas destacan 3 casos de sida.

Conclusión: Para realizar estudios estadísticos de mortalidad médica, es necesario contar con sistemas de registros adecuados. Se observó desde 1988 una tendencia creciente de defunción a edades menor de 10 años.

Se encontró un $10 \%$ de suicidios, lo que nos lleva a pensar no sólo en la salud física sino mental de nuestros médicos.

La principal causa de muerte entre los médicos fue la cardiovascular, destacando la enfermedad coronaria entre los médicos activos, por lo que urge llevar a cabo estrategias de intervención en factores de riesgo y estilos de vida saludable. (AMC, 1997;39 (2):38-43)

Descriptores: mortalidad, médico, tasas, servicios de salud.

\section{Introducción}

El médico, por su particular ejercicio profesional, está mís expuesto a la morbimortalidad de varias enfermedades, que otru tipo de profesionales. Esto no sólo para enfermedades físicos sino para enfermedades mentales como lo afirma un estudio británico', lo que afectará inevitablemente en su labor con los pacientes ${ }^{1}$.

Además, las Asociaciones Médicas no han actuado como grupos de soporte, pues no apoyan a los médicos que deben enfrentar demandas, actos que los predisponen a sufrir depresiones y suicidio'.

A lo anterior cabe agregar el efecto devastador de los factores do riesgo y los estilos de vida poco saludables, como producto de múltiples agresiones físicas y mentales en su ambiente laboral. con repercusiones en su ámbito familiar, económico y social. Las fuentes de estrés son numerosas y variarán dependiendo de la etapa de su carrera, circunstancias personales, especialidad y entorno laboral ${ }^{2}$.

Entre las causas más problemáticas de estrés se encuentran li sobrecarga de trabajo, el tipo de actividad, (atender al paciente. recibir llamadas, acudir a reuniones, horarios muy cambiantes. gran responsabilidad); tiempo insuficiente para concluir cl trabajo y también para descansar. Todo lo anterior producirá períodos crónicos exhaustivos de sobrecarga y agotamiento físico y mental'.

Duffy y Lidith describieron aleccionadoramente cómo un médico griego escribió para su futura lápida: "Estos son los deberes de un médico: primero la salud de su mente y darse ayuda a sí mismo. antes de ayudar a alguien más."

Los problemas del médico se han detectado desde que son estudiante o internos ${ }^{4}$, en donde la depresión, el enojo fácil y la fatiga son los problemas de salud mental más frecuentes.

Entre los médicos jóvenes cada vez existe más desmotivación como consecuencia del estrés y la depresión, producto de los frecuentes cambios de trabajo, domicilio incierto, alejamiento de sus familiares y problemas económicos ${ }^{2}$. Las largas jornadas laborales no son tanto el problema entre los internos y los residentes, sino la carencia de respaldo y de liderazgo, asociadas al temor de la inseguridad laboral ${ }^{5}$.

En términos generales, entre los doctores, de todas las edades, e indistintamente de los años de trabajo, cada vez hay un mayor

Dirección Regional de Servicios Médicos,

Región Chorotega

38 noviembre 1997, AMC, vol. 39 (2) 
grado de insatisfacción por haber elegido como carrera profesional la Medicina'.

Los problemas personales y laborales se magnifican debido a que, en nuestro continente, el desempleo de médicos aumenta constantemente?

Entre los médicos se encuentran numerosos matrimonios psicológicamente pobres, con problemas de abuso del alcohol, anfetaminas, somníferos o tranquilizantes ${ }^{8}$.

Los médicos sufren el doble de riesgo en accidentes de tránsito, tres veces más riesgo de suicidios y de cirrosis hepática y treinta veces más riesgo de adicción a las drogas, que la población en general'.

En Costa Rica, el Dr. Valverde Caravaca ${ }^{9}$ ha alertado acerca del hecho de que el médico muere relativamente joven: una década antes que el promedio de vida nacional, apenas cuando se está pensionando. Al morir, el promedio de edad de los galenos es de 63,3 años.

La presente investigación se llevó a cabo con el propósito de conocer las causas de muerte y el impacto de la profesión médica como factor de riesgo en el Cuerpo Médico Nacional, así como proponer estretagias para el abordaje de lo encontrado.

\section{Materiales y Métodos}

Jornadas de trabajo con la valiosa colaboración de distintas personas (ver nota de agradecimiento), durante el período de 1992 a 1994, en el Colegio de Médicos y Cirujanos, la Caja Costarricense de Seguro Social, el Ministerio de Salud, el Instituto Nacional de Seguros, el Registro Civil y conversaciones con colegas y familiares de colegas fallecidos.

En el Colegio de Médicos y Cirujanos se recopila información del Departamento de Archivo, directamente de los expedientes de los médicos fallecidos del primero de enero de 1982 al treinta de agosto de $1992^{10}$. Se tabula para cada caso el nombre completo, la edad al fallecer, la fecha del fallecimiento (día, mes y año), si era médico general o especialista y la fecha de nacimiento.

Del Departamento de Cómputo ${ }^{11}$ se recopila información sobre los médicos activos, pensionados, ausentes, residentes y en servicio social obligatorio, con corte al treinta y uno de diciembre de 1991.

En ciertos casos se complementaron los datos solicitando información directamente a familiares de colegas fallecidos, incluyendo entre estos algunos colegas.

En el Registro Civil se realizaron jornadas de trabajo asistidas en la Sección de Archivo, investigando nombres completos y números de certificados de defunción de todos los médicos fallecidos del primero de enero de 1982 al treinta de agosto de $1992^{12}$, para luego trabajar en la Oficina de Defunciones, analizando todos los certificados de defunción de los colegas ${ }^{13}$. En estas jornadas de trabajo se corroboraron el año del fallecimiento, el lugar, las causas primarias y secundarias de defunción y la edad de los médicos al momento de morir. En la Caja Costarricense de Seguro Social se consultó sobre la cantidad de plazas para médicos (a diciembre de 1991) y el número de médicos que laboran para la institución ${ }^{14}$.

En el Ministerio de Salud e Instituto Nacional de Seguros se consultó sobre la cantidad de médicos que laboran para estas instituciones de salud, a diciembre de $1991^{15,16}$.
TABLA 1

Cuerpo Médico Nacional, según actividad. Costa Rica, 1991 (números absolutos y porcentuales)

\begin{tabular}{lrr}
\hline Condición de actividad & Absoluto & $\%$ \\
\hline $\begin{array}{l}\text { Médicos activos } \\
\text { Médicos Generales }\end{array}$ & 1.425 & 36,6 \\
$\quad$ Médicos Especialistas & 1.698 & 43,6 \\
Médicos pensionados & & \\
$\quad$ Médicos Generales & 65 & 1,67 \\
$\quad$ Médicos Especialistas & 201 & 5,16 \\
Médicos ausentes & & \\
$\quad$ Médicos Generales & 186 & 4,78 \\
$\quad$ Médicos Especialistas & 97 & 2,49 \\
Médicos servicio social & 146 & 3,75 \\
Médicos residentes extranjeros & 76 & 1,95 \\
$\quad$ Total & 3,894 & 100,0 \\
\hline Fuente: Departamento de Cómputo, Colegio de Médios
\end{tabular}

Fuente: Departamento de Cómputo, Colegio de Médicos y Cirujanos de Costa Rica.

El Procesamiento de Datos se realizó por medio de programas computarizados Word Perfect (versión 5.1), Quattro Pro, DBase IV Plus y Harvard Graphics (3.0).

Esta investigación se presentó como trabajo libre durante el LIII Congreso Médico Nacional (San José, diciembre de 1992). galardonándose con el premio al mejor trabajo libre presentado.

\section{Resultados}

En Costa Rica, a diciembre de 1991 había 3894 médicos, casi todos activos $(80 \%)$ y muy pocos pensionados $(6,8 \%)$. Li mayoría de los activos eran especialistas $(43,6 \%)$ y entre estos los más numerosos eran pediatras generales $(15,1 \%)$, internistas $(13,4 \%)$ y gineco-obstetras $(11,2 \%)$. (Tabla 1 )

De acuerdo con el sexo, la mayoría del cuerpo médico estaba compuesta por hombres $(76,3 \%)$, con una de proporción 3:1 con respecto a las mujeres, la cual que se reduce a 1,5:1 en las generaciones menores a los cuarenta años de edad.

Sobre los grupos etarios, el $41,6 \%$ del cuerpo médico nacional era menor a los cuarenta años de edad (Tabla 2). Nacional, según grupos etarios y sexo. Costa Rica, 1991. (números absolutos y porcentuales)

\begin{tabular}{c|r|r|r|r}
\hline $\begin{array}{c}\text { GRUPOS ETARIOS } \\
\text { (en años) }\end{array}$ & \multicolumn{2}{|c|}{ DOCTORAS } & \multicolumn{2}{c}{ DOCTORES } \\
Absoluto & \multicolumn{1}{c}{$\%$} & Absoluto & \multicolumn{1}{c}{$\%$} \\
\hline TOTAL & 924 & 100,0 & 2.970 & 100,0 \\
$20-29$ & 220 & 23,8 & 344 & 11,6 \\
$30-39$ & 530 & 57,4 & 1.091 & 36,7 \\
$40-49$ & 112 & 12,1 & 643 & 21,6 \\
$50-59$ & 46 & 5,0 & 562 & 19,0 \\
60 y más & 16 & 1,7 & 330 & 11,1 \\
\hline
\end{tabular}
Fuente: Departamento de Cómputo, Colegio de Médicos y Cirujanos de
Costa Rica 
TABLA 3

Cuerpo Médico Nacional y relación de defunciones, sexo y grupos etarios. Costa Rica, 1982 a 1992

(en números absolutos)

\begin{tabular}{|c|c|c|c|c|c|c|}
\hline \multirow[t]{2}{*}{$\begin{array}{l}\text { EDADES } \\
\text { (años) }\end{array}$} & \multicolumn{3}{|c|}{$\begin{array}{l}\text { Cuerpo Médico Nacional } \\
\text { (a diciembre de 1991) }\end{array}$} & \multirow[b]{2}{*}{ Fem. } & \multirow{2}{*}{$\begin{array}{c}\text { Médicos fallecidos } \\
1982-1992^{*} \\
\text { Masc. }\end{array}$} & \multirow{2}{*}{ Total } \\
\hline & Fem, & Masc. & Total & & & \\
\hline TOTAL & 924 & 2.970 & 3.894 & 6 & 113 & 119 \\
\hline $20-29$ & 220 & 344 & 564 & 1 & 2 & 3 \\
\hline $30-39$ & 530 & 1.091 & 1.621 & 1 & 11 & 12 \\
\hline $40-49$ & 112 & 643 & 755 & 0 & 14 & 14 \\
\hline $50-59$ & 46 & 562 & 608 & 1 & 19 & 20 \\
\hline 60 y más & 16 & 330 & 346 & 3 & 67 & 70 \\
\hline
\end{tabular}

- Del primero de enero de 1982 al treinta de agosto de 1992 Fuentes: Colegio de Médicos y Cirujanos y Registro Civil

Según el lugar de residencia, la mayoría vivía en la provincia de San José $(66,2 \%)$, Heredia era la provincia en donde vivía la menor cantidad de médicos generales, mientras que en las zonas rurales, fuera de San José, vivía el número más pequeño de especialistas. No puede afirmarse que el lugar de residencia sea sinónimo del lugar de trabajo.

Desde el punto de vista de trabajo institucional, el ochenta y ocho por ciento de los médicos costarricenses trabajaban en la Caja Costarricense de Seguro Social jornada completa o incompleta, y en el Ministerio de Salud el diez por ciento.

Según el lugar de formación, la mayoría de los médicos se graduaron en las dos universidades nacionales $(58,9 \%)$. Sobre los médicos formados en el exterior, los países de mayor aporte fueron México $(n=751)$, España $(n=170)$ y Argentina $(n=124)$. Como hallazgo fortuito, pero relevante se encontró que entre los médicos pensionados hay setenta colegas que nunca cotizaron para el Fondo de Auxilio y Retiro Médico, viviendo actualmente con pensiones muy reducidas y en condiciones socioeconómicas muy difíciles. Se contactó con la Junta de Gobierno de nuestro colegio profesional, con el fin de analizar qué podríamos hacer por ellos.

Se investigaron las causas de defunción de los colegas fallecidos del primero de enero de 1982 al treinta de agosto de 1992 Fallecieron ciento diecinueve médicos, la mayoría especialistas $(n=67: 56,4 \%)$ y entre estos, mayormenteente gineco-obstetras $(n=13)$ y pediatras $(n=10)$. Solo seis doctoras murieron, todas ellas médicas generales.

Se muestra el desglose según grupos etarios del cuerpo médico nacional y las defunciones encontradas en el período estudiado, pero no se trabajan tasas, por tratarse de poblaciones en distintos períodos (Tabla 3 ).

La mayor cantidad de médicos falleció entre los sesenta y setenta y nueve años de edad (40\%). (Tabla 3)

A partir de 1988 se observa una tendencia creciente de defunciones a edades menores,durante estos muchos médicos estaban aún trabajando o se habían pensionado recientemente (Figura 1).

La mayoría de las causas de muerte fueron cardiovasculares $(n=46 ; 39,2 \%)$ y entre estas destacaron diecinueve infartos agudos al miocardio $(n=11$ de los cuales, el $54,5 \%$ se produjo

entre los médicos activos). El mayor número de estos infartos se presentó en los últimos tres años.

El segundo grupo más frecuente fueron los carcinomas $(n=2+$ : $20,5 \%$ ), en donde se documentan diecisiste tipos diferentes do cáncer, casi todos entre los médicos activos y menores de sesentil y cinco años de edad (57\%).

El tercer grupo de enfermedades (otras) incluye efectos del etilismo, problemas gastrointestinales, nefropatías, etc. Al analizar las causas en este grupo, se produce la sensación de que hubo mucha enfermedad encubierta (por ejemplo, miocardiopatía dilatada más cirrosis hepática pudieron ser causa de etilismo). Entre las causas médico legales $(n=12 ; 10 \%)$ destaca la predominancia de suicidios y, por lo menos, en una defunción se demostró la mala praxis como causal directa de esta decisión del colega. Otro médico se suicidó al enterarse de que padecía de sida. Había también accidentes automovilísticos (uno de ellos en la Guerra del Golfo), un ahogado y un accidente laboral.

Entre las causas infecciosas $(n=11 ; 9,4 \%)$ destacan tres casos de sida, todos en menores de cuarenta y cinco años de edad y diagnosticados durante los últimos tres años. La bronconeumonía fue la causa infecciosa más frecuente de muerte entre los médicos seniles.

El grupo se ignora (Figura 2$)(n=9 ; 7,7 \%)$ corresponde a colegas que fallecieron en el exterior, y de los que en nuestro país, ni el Registro Civil ni el Colegio de Médicos y Cirujanos poseen información sobre las causas de fallecimiento. La mayoría eran médicos mayores de sesenta y cinco años de edad y que habían fallecido cinco o más años atrás.

Durante el año de 1982 fallecieron once médicos, nueve de los cuales eran igual o mayores a los sesenta y cinco años de edad. Conforme transcurre la década estudiada, la proporción de fallecimientos "médicos activos/médicos pensionados" se mantenía mayor en el denominador, para igualarse y luego invertirse en el año de 1990 (Figura 1).

\section{Discusión}

En 1975, Laeger y Aerzte propusieron en un estudio comparativo de esperanza de vida y mortalidad entre médicos y población general, realizado simultáneamente en Alemania, Dinamarca y las Filipinas, que la profesión médica era un factor de riesgo para 
FIGURA 1

Médicos fallecidos 1982-92, según edad y año de muerte.

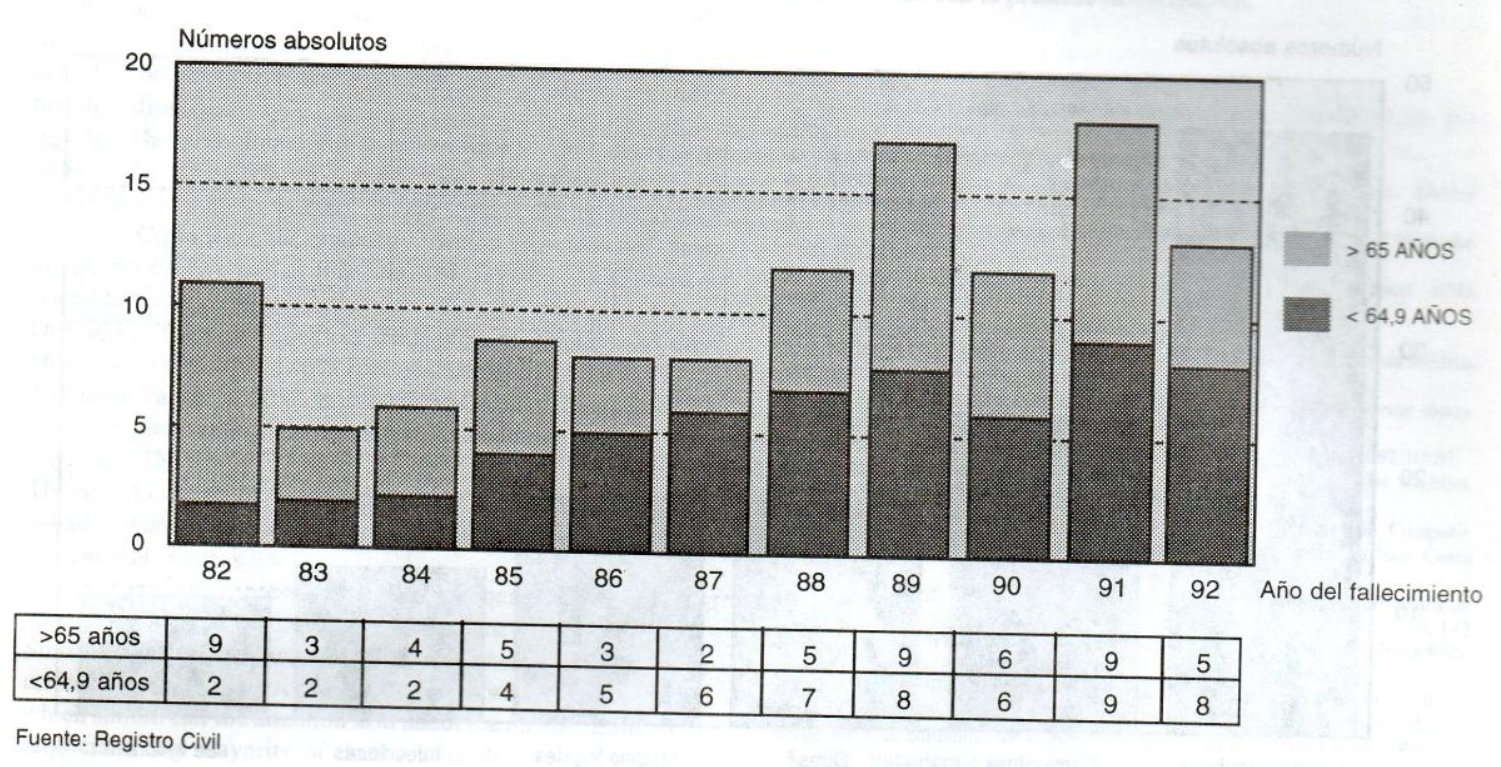

morir joven ${ }^{17}$.

La presente investigación es la primera que se realiza en el nivel nacional, sobre la mortalidad en el cuerpo médico nacional. Hemos observado lo necesario y debemos formular también la hipótesis de que en Costa Rica, la Medicina como profesión, es riesgosa, para posteriormente deducir y verificar lo necesario con el fin de profundizar en el impacto que la profesión médica tiene sobre la calidad de vida entre los médicos costarricenses.

Tal vez Esculapio lo que también debió escribir entre sus consejos fue: "si quieres morir antes, hazte médico, hijo mío".

Tal y como se ha investigado en otros países ${ }^{18}$, este estudio pretende llamar la atención sobre el estado del proceso salud y enfermedad en el cuerpo médico nacional.

De acuerdo con el Dr. Valverde Caravaca, la tasa de mortalidad general en Costa Rica es de 4,6 por mil habitantes, mientras que la de los médicos es de 3,7 por mil habitantes ${ }^{9}$

Si bien cada vez hay más médicos en el país, la tendencia de muchos galenos a morir cuando están finalizando sus años de labor institucional, o a escasos años de haberse pensionado, debe considerarse preocupante, ya que se supone que la condición socioeconómica del médico costarricense debería coadyuvar a que el médico viva más años y con una buena calidad de vida. Adicionalmente, si esto está sucediendo con los médicos costarricenses, me pregunto si esta tendencia se manifestará de manera similar con otros profesionales, en nuestro país.

Con el presente estudio no se puede concluir que los médicos costarricenses tengan una menor espectancia de vida que la nacional ya que tendríamos que elaborar estudios prospectivos comparando las causas de muerte por grupos de edad, con otros grupos de profesionales y no profesionales, así como ajustar las tasas de mortalidad por grupos etarios y sexo, y compararlas con otros grupos de costarricenses.

Paralelamente a reconocer la limitación anterior, considero que el cuerpo médico nacional no debe esperar los resultados de investigaciones más profundas sobre su mortalidad y su morbilidad. Debemos hacer algo al respecto lo más pronto
posible.

Es bien conocida la predisposición de los médicos a morir por infarto agudo del miocardio ${ }^{19}$. Sin embargo, llama la atención la tendencia entre los médicos de que se presenten cada vez a menor edad.

Becker demostró en los Estados Unidos de Norteamérica, que entre las poblaciones de mayor riesgo para padecer el alcoholismo como enfermedad, se encuentran los médicos ${ }^{25}$.

Entre las causas primarias y secundarias descritas en los certificados de defunción se leen muchos cuadros que pudieron tener una relación con el etilismo como causal.

Entre los colegas fallecidos hubo siete suicidios, todos entre médicos activos, en una muestra de ciento diecinueve médicos. Esto debe llamar a la reflexión.

Pilowski y O'Sullivan recomiendan campañas dirigidas a los médicos, que orientan a cambios en sus actitudes con respecto a su profesión, sus pacientes y su entorno familiar'. Afirman que es importante inculcar en los médicos la necesidad de tener amigos y de que busquen apoyo en sus familiares.

$\mathrm{Al}$ abordar temas tan delicados como la drogadicción entre los médicos, debemos reconocer que los colegas están más predispuestos a las drogas, en parte por la facilidad con que 
FIGURA 2

Causas primarias de muerte. Cuerpo Médico Nacional, 1982-1992

Números absolutos

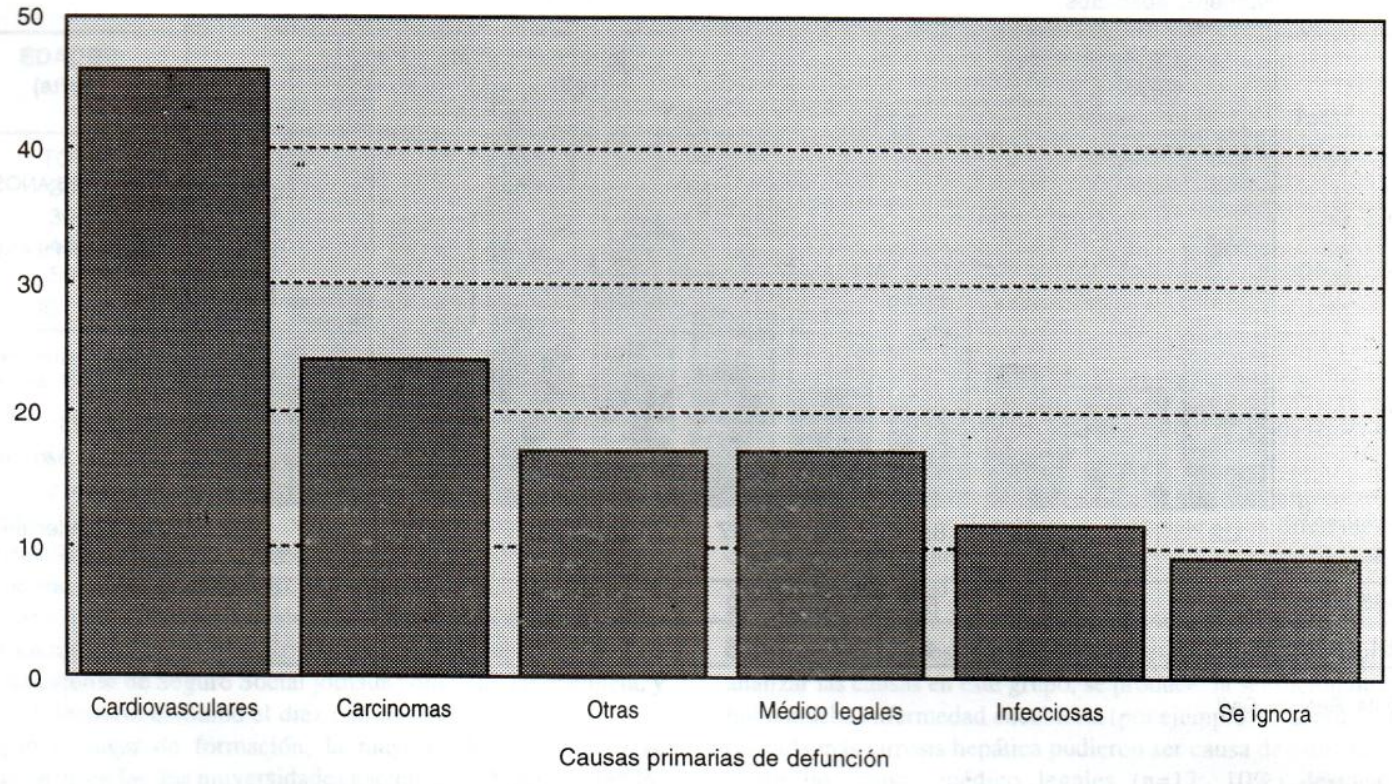

Fuente: Registro Civil

pueden conseguirlas ${ }^{21}$, y debido a que su rehabilitación se dificulta, es importante desarrollar estrategias de educación orientados a estos profesionales.

El hallazgo fortuito, relacionado con los médicos pensionados, puede ser una señal de alarma de las difíciles condiciones socioeconómicas que muchos médicos podrían padecer si no preveen bienes y pensiones adecuadas que les permitan vivir cómodamente, después de toda una vida de servicio institucional. Para investigar la morbilidad y la mortalidad entre los médicos, nos encontramos a la fecha de cierre de este estudio con varias limitantes : las instituciones de salud y el Colegio de Médicos y Cirujanos no tienen registros anuales de la cantidad de médicos de Costa Rica.

En otras palabras, a 1992 no podemos estimar tasas de mortalidad comparando la población actual de médicos, por ejemplo, con el registro de defunciones de los últimos diez años, ya que incurriríamos en un error estadístico al comparar poblaciones de diferentes períodos y si nos atreviésemos a hacerlo, el error estribaría paralelamente en que, entre 1982 y 1992, el crecimiento de la población médica ha tenido una progresión geométrica, por lo que las tasas espacíficas y las ajustadas no deberían calcularse.

Para investigar la morbilidad y la mortalidad del Cuerpo Médico Nacional debemos solicitarle al Colegio de Médicos de Cirujanos y a nuestras Instituciones de Salud, que manejen un sistema de información uniforme de estadísticas básicas para conocerse con la exactitud necesaria cuántos médicos hay en el país. sus condiciones laborales y personales.

Es urgente llevar a cabo un estudio sobre la morbilidad en el cuerpo médico nacional. Tenemos que analizar los factores de riesgo, los cambios en los estilos de vida, el estado de las jornadas laborales (sobre todo las institucionales); en fin. visualizar científicamente la problemática de los médicos costarricenses.

Los resultados del presente trabajo, así como la propuesta de un estudio de morbilidad, deben compartirse con todos los médicos. para llevar a cabo acciones a distinto nivel (educativas, cambios laborales, salud pública) con el Colegio de Médicos y Cirujanos. las Instituciones de Salud, los Sindicatos Médicos y muy importante: todos nosotros, médicos que tenemos una profesión que se insinúa como factor de riesgo para morir prematuramente a causa de muchas patologías, la mayoría de ellas controlables con medidas preventivas y cambios de actitudes.

\section{Abstract}

Justification: Medical profesionals are predisposed subject to early appearence of morbi-mortality caused by various diseases as a result of their work. This fact must be verified in our country.

Objetive: Investigation of mortality in Costa Rica in the last eleven years by means of a descriptive, transverse study. Methods: This is a revison of characteristics of the laboral

42 noviembre 1997, AMC, vol. 39 (2) 
activity, sex, age, residency, country of studies and speciality of living physicians, and also the death causes, age at time of death and the cause of death of physicians thar died from January 1982 to august 1992, acording to registries from the Costa Rica's colledge of physicians and surgeons, civil registry and three health Institutions.

Results: The study found that in Costa Rica in 1991, there were 3894 doctors, $43.6 \%$ of whom were specialists, in a $3: 1$ proportion of men to women, $58.9 \%$ studied and recieved their degres in Costa Rica, the majority of which were under 40 years of age. $66.2 \%$ live in San Jose and $80 \%$ work for the Costarican Social Security System.

During the study, 119 doctors died, $95 \%$ of whom were men, $56.4 \%$ were specialists, $40 \%$ between the ages of 60 and 79 .

The main cause of death was cardiovascular diseases $(39.2 \%)$, leing coronary antery disease the most important within active physiciam (54.5\%).

The second cause of death was cancer with $20.5 \%-10 \%$ of suicides were found and among infections causes, AIDS was present with 3 cases

Conclusion: It is necessary to have adequate registers to do mortality statistical studies of the physicians.

Since 1988 an increasing mortality was observed in the group under 60 years. Ten percent of the deaths were due to suicides, which should call the attention also about the mental health of the physicians The mayority of deaths were due to cardiovascular disease among the practicing physicians which show to need to develop strategies to control risk factors and stimulate healthy living habits.

\section{Reconocimientos}

Al Sr. Mario Loaiza Jiménez (Administrador) al Sr. Alvaro Fernández Gómez (Departamento de Cómputo y a la Srita. Rosa I. Castro (secretaria, todos del Colegio de Médicos y Cirujanos. A la Licda. Marisol Castro Dobles (Directora General), al Sr. Asdrúbal Molina (Jefe Oficina de Defunciones) y al Sr. Eduardo Gómez A. (Sección de Archivo), todos del Registro Civil. Al Sr. Reinaldo Arias Camacho, Ministerio de Salud. Al Sr. José R. Orozco, Instituto Nacional de Seguro Social.
A todos ellos muchas gracias por su apoyo, comprensión y colaboración con la presente investigación.

\section{Referencias}

G. Mental Illness in Doctors. BMJ, 1989:298: 269270.

Lask B. Forget the stiff upper lip. BMJ, 1987;295:1584-85.

3. Duffy J, Litih E. Psychiatric morbidity on physicians. JAMA. 1964;189:989-992.

4. Lynn W. The Internship and Health Problems. Western Journal of Medicine. 1986;144: 93-98.

5. Stress in Junior Doctors : stress and support. BMJ. 5. Dudley H. 1990 .

1990;301:75-76.

6. Lowry S. Dr. Kildare let me down. BMJ, $1988 ; 296$ : 1686-87. 7. Malagón

8. Vaillant G, Brighton J, McArthur C. Physicians use mood-altering drugs. New England Journal of Medicine, 1970;270:365-370

9. Valverde C. La Mortalidad en los Médicos. Opinión Médica, 1992:10:20.

10. Colegio de Médicos y Cirujanos de Costa Rica. Sección de Archivo 10. Colentes de Médicos Fallecidos. San José, Costa Rica, 1992.

Expedientes de Médicos y Cirujanos de Costa Rica. Oficina de Cómputo

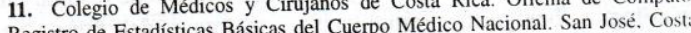
Registro de
Rica 1992.

Rica 1992. 12. Registro Civil. Sección de Archivo. Libros de Defunciones y Registro de Archivo de Certificados de Defunción. Libros 1 ( 340 al 384), 2 (191), 3 (139, 142 y 143), 4 (078), 5 (081, 083 y 085) y $6(087,089$ y 090). San José, Costa Rica.

13. Registro Civil. Oficina de Defunciones. Certificados de Defunción de los Médicos Fallecidos 1982 a 1992. La copia de los números de certificados defunción y su contenido los conserva el autor. San José, Costa Rica. 1992. defunción y su contenido los conserva el autor. Saja costarricense de Seguro Social. Dirección de Recursos Humanos. 14. Caja costarricense de Seguro Social. Oficinas Centrales. San José. Costa Rica. Registr
1992.
15. M

15. Ministerio de Salud. Sección Clasificación y Valoración. Planillas de Trabajadores. Oficinas Centrales, San José, Costa Rica, 1992.

16. Instituto Nacional de Seguros. Departamento de Personal. Unidad de Servicio al Personal. Oficinas Centrales, San José, Costa Rica, 1992

17. Deutsche Statistic Aertze, Medizinische Danske Laeger, National Center for 17. Deutsche Statistic Aertze, Meor Phillipines y Mortimer Spiegelman. Las dealth Statistics fel electrocardiograma. Médico Moderno, 1975;13:11-17.

denuncias del electrocardiogram. Méderno 1975:13:18

29.

19. Stampfer, Malinow M.R, Willwt W.C. A Prospective Study of Plasma Homocysteine and Risk of Miocardial Infarction in US Physicians. JAMA. $1992 ; 268: 877-881$.

20. Becker Ch. Alcohol and Drug Abuse - Is there a safe amount? Wester! Journal of Medicine, 1984;141:884-890.

21. Farnsworth D. Drug dependence among physicians. New England Journal of Medicine, 1970;271:392-393.

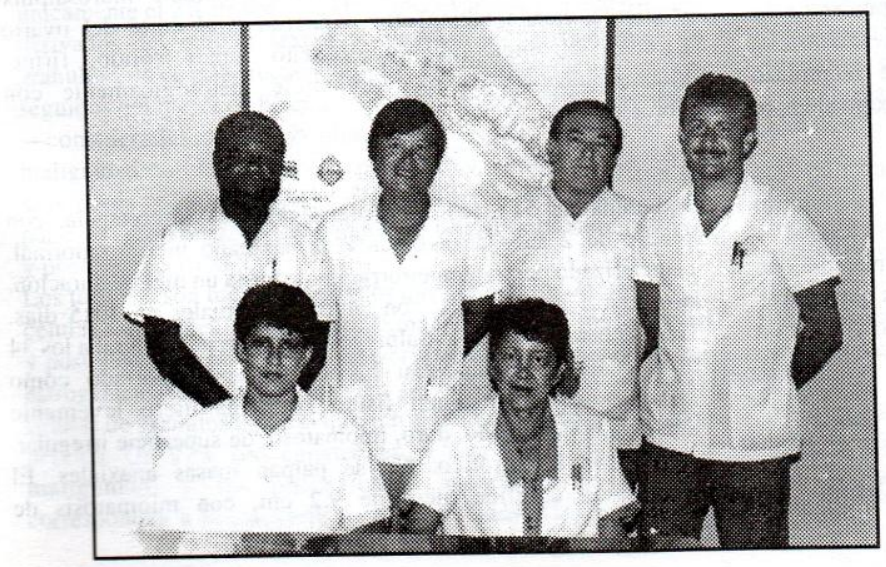

Cuerpo Médico de la Dirección Regional Chorotega 